\title{
Empirical multi-wavelength prediction method for Very High Energy Gamma-ray emitting BL Lacs
}

\author{
V. Fallah Ramazani ${ }^{1}$, E. Lindfors ${ }^{1}$ and K. Nilsson ${ }^{2}$ \\ ${ }^{1}$ Tuorla Observatory, Department of Physics and Astronomy, University of Turku, Finland \\ ${ }^{2}$ FINCA (Finnish Center for Astronomy with ESO), University of Turku, Finland
}

\begin{abstract}
We present the most up-to-date and complete multi-wavelength correlation analysis on luminosity properties of TeV BL Lacs. Correlation function (power law or linear) parameters are calculated based on linear regression method. Using the lower energy luminosities of a sample of 182 non-TeV BL Lacs and the generated functions, minimum level of VHE gammaray emission was calculated for each non-TeV BL Lacs. This multi wavelength prediction method gives us a list of best candidates to be observed with current generation of Imaging Air Cherenkov Telescopes.
\end{abstract}

Keywords. BL Lacs, VHE gamma-ray

\section{Introduction}

The majority of extragalactic objects detected in Very High Energy (VHE) $\gamma$-ray band $(>100 \mathrm{GeV})$ are from rare type of radio-loud Active Galactic Nuclei (AGN) called BL Lacs. As a subclass of blazars, BL Lacs' jets are nearly aligned to the observer's line of sight. As bright distant objects, VHE $\gamma$-ray emitting $(\mathrm{TeV}) \mathrm{BL}$ Lacs can be used to study the universe between us and BL Lacs. The observed properties of BL Lacs in VHE $\gamma$-rays band are measured using targeted observation by Imaging Air Cherenkov Telescopes (IACTs). Only 57 BL Lacs are detected in VHE $\gamma$-rays band. Therefore, IACTs are expected to discover many BL Lacs in VHE gamma-rays band. But due to the restrictions on current technology a prediction method based on the lower energy data is demanding. We constructed a prediction method based on the cross bands luminosity of TeV BL Lacs.

\section{Samples, Data and methodology}

Radio (4.85 GHz), Optical (R-band), X-ray (2-10 keV) and Gamma-ray (1-100 GeV) data are collected from literature and databases for $\mathrm{TeV}(\# 47)$ and non-TeV (\#182) BL Lacs. VHE $\gamma$-ray flux $(>200 \mathrm{GeV})$ are collected for TeV sample by dividing this sample to multiple (Group A) and single detected (Group B). Five VHE $\gamma$-ray data sets are formed based on different states of group A (high state and low state), group B and combination of group B with each state of group A. Figure 1 illustrate the distribution of $\mathrm{TeV}$ sample in different Luminosity-Luminosity planes in logarithmic scale. Figure 2 illustrates the approach to check the existence of correlation and calculating the best fitted model parameters. The lower energy band luminosities of non-TeV BL Lacs are used as variable input for the prediction functions. We calculate at least 4 levels of VHE gamma-ray luminosity for each non-TeV BL Lac. The non-TeV BL Lacs with highest predicted VHE gamma-ray flux are selected as the promising TeV candidates. 


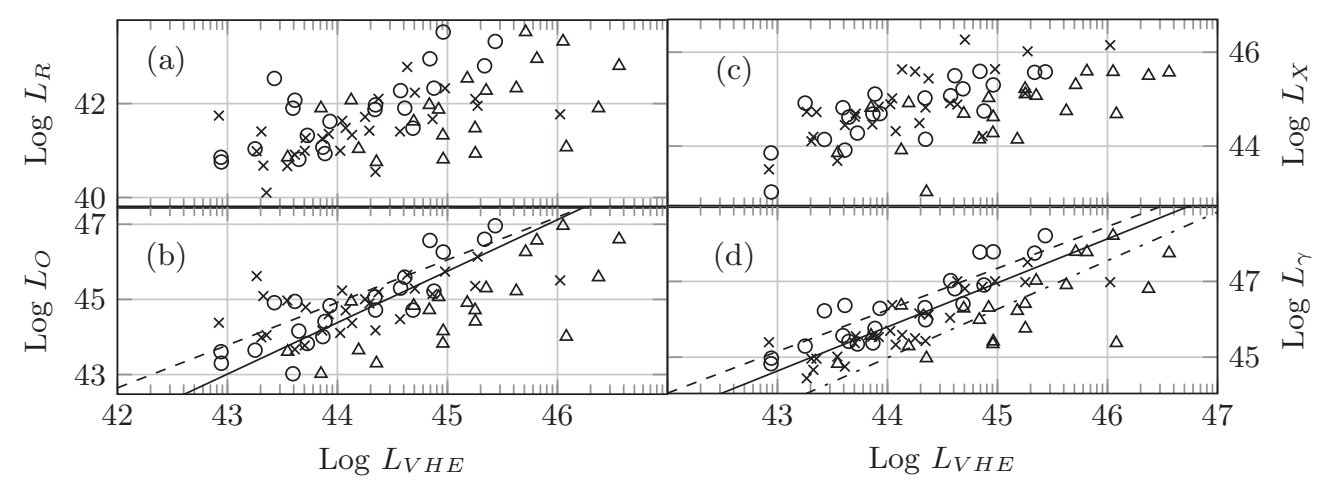

Figure 1. Luminosity in the VHE $\gamma$-ray band versus the luminosity in radio (a), optical (b), $\mathrm{X}$-ray (c) and $\gamma$-ray (d) bands in logarithmic scale. All the axis are in [erg/s] unit. The different symbols represent the data of different groups (see text): Low state group A (circle), High state group A (triangle) and group B (cross). The correlation functions of the low state of group A is shown with dashed line. The solid/dot-dashed lines represent the correlation functions of the combined data sets of group B and low/high state of group A.

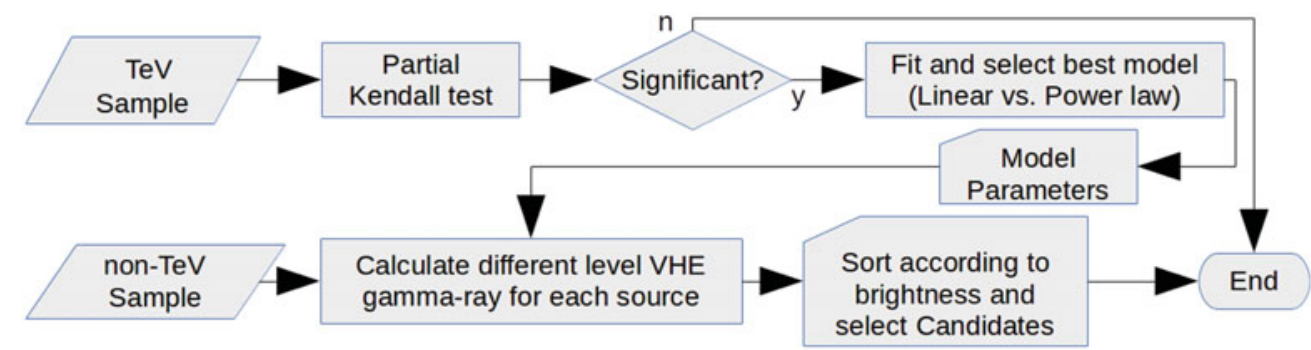

Figure 2. Statistical approach for selecting TeV BL Lacs Candidates.

Table 1. Promising TeV BL Lacs candidates. The fluxes are in $\left(\mathrm{erg} / \mathrm{cm}^{2} / \mathrm{s}\right)$ unit.

\begin{tabular}{lcccc}
\hline Source name & $\mathrm{z}$ & $S_{V H E, \text { min }}$ & $S_{V H E, \text { med }}$ & $S_{V H E, \text { max }}$ \\
\hline PMN J0444-6014 & 0.097 & $5.40 \times 10^{-12}$ & $1.71 \times 10^{-11}$ & $2.31 \times 10^{-11}$ \\
3C 371 & 0.046 & $5.29 \times 10^{-12}$ & $3.37 \times 10^{-11}$ & $1.79 \times 10^{-10}$ \\
B2 1811+31 & 0.117 & $4.96 \times 10^{-12}$ & $9.41 \times 10^{-12}$ & $2.11 \times 10^{-11}$ \\
1RXS J195815.6-301119 & 0.119 & $3.33 \times 10^{-12}$ & $1.06 \times 10^{-11}$ & $1.71 \times 10^{-11}$ \\
1H 1914-194 & 0.137 & $3.04 \times 10^{-12}$ & $9.37 \times 10^{-12}$ & $2.95 \times 10^{-11}$ \\
PKS 0829+046 & 0.174 & $2.98 \times 10^{-12}$ & $1.60 \times 10^{-11}$ & $3.86 \times 10^{-11}$ \\
RBS 0351 & 0.265 & $2.53 \times 10^{-12}$ & $1.47 \times 10^{-11}$ & $2.57 \times 10^{-11}$ \\
B2 0806+35 & 0.083 & $2.49 \times 10^{-12}$ & $5.17 \times 10^{-12}$ & $1.32 \times 10^{-11}$ \\
PMN J1936-4719 & 0.265 & $2.34 \times 10^{-12}$ & $5.47 \times 10^{-12}$ & $4.96 \times 10^{-11}$ \\
\hline
\end{tabular}

\section{Results and Conclusions}

There are 7 significant correlations between lower energy bands and TeV band. All the significant correlations can be described better with power law model. We build our prediction functions based on these correlations. The lower energy band luminosities of non-TeV BL Lacs are used as variable input for the prediction functions. At least 4 levels of VHE gamma-ray luminosity are calculated for each non-TeV BL Lac. The non-TeV BL Lacs with highest predicted VHE gamma-ray flux are selected as the promising TeV candidates and listed in table 1. 